\title{
Cryogenic $Q$-factor measurement of optical substrates for optimization of gravitational wave detectors
}

\author{
S Nietzsche ${ }^{1}$, R Nawrodt ${ }^{1}$, A Zimmer ${ }^{1}$, R Schnabel $^{2}$, W Vodel $^{1}$ and \\ P Seidel $^{1}$ \\ ${ }^{1}$ Institut für Festkörperphysik, Friedrich-Schiller-Universität Jena, Helmholtzweg 5, \\ D-07743 Jena, Germany \\ ${ }^{2}$ Max-Planck-Institut für Gravitationsphysik, Universität Hannover, Callinstraße 38, \\ D-30167 Hannover, Germany \\ E-mail: Sandor.Nietzsche@uni-jena.de
}

Received 13 October 2005, in final form 1 December 2005

Published 16 March 2006

Online at stacks.iop.org/SUST/19/S293

\begin{abstract}
Future generations of gravitational wave interferometers are likely to be operated at cryogenic temperatures because one of the sensitivity limiting factors of the present generation is the thermal noise of end mirrors and beam splitters that occurs in the optical substrates as well as in the dielectric coatings. A possible method for minimizing thermal noise is cooling to cryogenic temperatures, maximizing the mechanical quality factor $Q$, and maximizing the eigenfrequencies of the substrate. We present experimental details of a new cryogenic apparatus that is suitable for the measurement of the temperature-dependent $Q$-factor of reflective, transmissive as well as nano-structured grating optics down to $5 \mathrm{~K}$. In particular, the SQUID-based and the optical interferometric approaches to the measurement of the amplitude of vibrating test bodies are compared and the method of ring-down recording is described.
\end{abstract}

(Some figures in this article are in colour only in the electronic version)

\section{Introduction}

Gravitational wave detectors are amongst the most sensitive devices ever built. The currently operating detectors LIGO and GEO 600 [1] now reach the noise spectral densities for a strain measurement of $5 \times 10^{-23} \mathrm{~Hz}^{-1 / 2}$ and $7 \times$ $10^{-22} \mathrm{~Hz}^{-1 / 2}$, respectively. Upcoming generations of interferometric gravitational wave detectors with even better sensitivities - currently in the design state-will presumably benefit from cryogenic techniques to reduce the thermal noise of optical components. Currently a number of different substrate materials such as sapphire, calcium fluoride, and silicon in combination with dielectric coatings and nanostructured surfaces are being considered [2]. The goal is to find a combination that maximizes the mechanical quality factor $Q$ and the eigenfrequencies at some realistic cryogenic temperature [3]. It is therefore essential to measure the temperature dependence of the two quantities mentioned for a large variety of mirror substrates. Nano-structured optics comes into play because future gravitational wave interferometers will store laser powers of several megawatts to reduce photon shot-noise. The combination of both cryogenic temperature and high laser power is a technical challenge. Even the highest grade fused silica which is used as test mass material shows a residual absorption of the order $1 \mathrm{ppm} \mathrm{cm}^{-1}$. Optics that transmits laser light is therefore heated. As a consequence the transmissive optics in high power interferometers cannot be held at its cryogenic operation point. Current research is investigating how beam splitters and cavity couplers can be replaced by reflective diffraction gratings based on nano-structured optics $[4,5]$. The influence of nano-structures on the quality factor is still not understood. The new cryogenic apparatus described here is in line with the demand for new experimental data. 


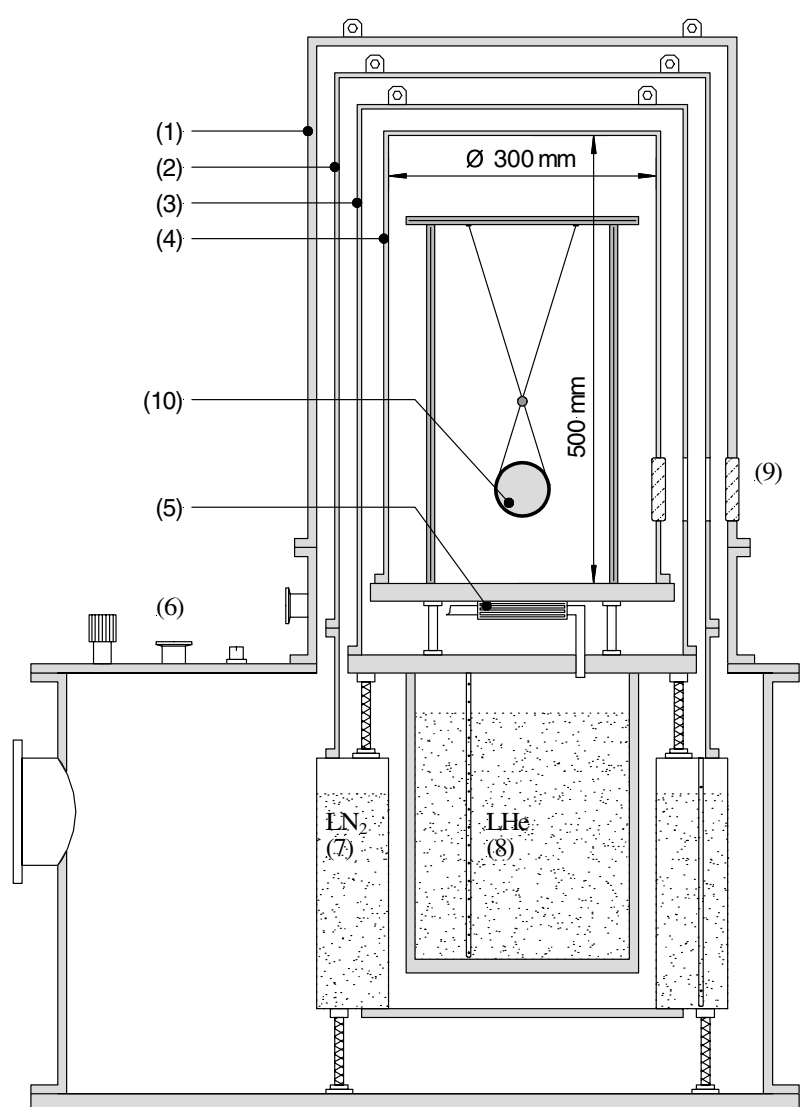

Figure 1. Schematic view of the cryostat providing 4.2-300 K at the probe volume. The probe volume (4) with a diameter of $300 \mathrm{~mm}$ and a height of $500 \mathrm{~mm}$ is cooled by a heat exchanger (5). It is surrounded by a $4.2 \mathrm{~K}$ shield (3) and a $77 \mathrm{~K}$ shield (2). Optical windows (9) in all shields allow the use of optical interferometers for read-out. The LHe (8) and $\mathrm{LN}_{2}$ (7) tanks are placed below the experimental platform. Feedthroughs (6) for sensors and the contact gas are available. The test sample (10) is suspended by a tungsten wire loop as a pendulum.

\section{Experimental set-up}

Figure 1 shows the schematic view of our new cryogenic apparatus allowing the measurement of the temperature dependence of the $Q$-factor in the range $5-300 \mathrm{~K}$. It provides a He vapourcooled probe chamber housing the pendulum suspended test body. For lowering the heat entry into the probe volume it is surrounded by two heat-radiation shields, one connected to the liquid helium tank and one to the liquid nitrogen tank. All shields and vacuum chambers are equipped with an optical window for an interferometric vibration read-out. For maintenance and test sample exchange all shields can be removed.

The probe volume is placed above the cryogenic tanks. This configuration enables the operator to build up easily different suspension systems at the cold plate to investigate the influence of the suspension system (e.g. wire length, material, diameter) on the measured $Q$-factor. Also, the excitation and read-out system can be fully adjusted before closing the cryostat.

The probe volume is cooled by the boil-off gas from the liquid helium tank using a heat exchanger at the bottom of the experimental platform. The helium gas flow is adjustable to control the cooling power. This technique provides low mechanical disturbances, which is an important issue due to the small vibration amplitudes to be measured. Another advantage of this design is that the boil-off rate of the helium tank is independent from the probe temperature.

The test sample is suspended as a pendulum inside the probe volume using tungsten wire with a diameter of $50 \mu \mathrm{m}$. This provides a low loss suspension and an excellent decoupling from outer mechanical disturbances.

To change the sample's temperature helium gas with a residual pressure of about $100 \mathrm{~Pa}$ is applied to the probe volume. The probe volume temperature is controlled by a LakeShore 340 temperature controller [6]. For thermometry a second test sample (identical in shape and material) is placed in the probe volume. A silicon diode is attached to the second test body to measure its temperature. After thermal equilibrium is reached the probe volume is evacuated. With a vacuum better than $10^{-2} \mathrm{~Pa}$ a temperature drift of lower than $200 \mathrm{mK} \mathrm{h}^{-1}$ was achieved.

The substrate is excited to its natural vibrations by the use of an electrostatic meander shaped exciter. By applying a high ac voltage to the meander strips an alternating electrical field is generated. This field generates an exciting force inside of the test body.

Currently we use a Michelson-like interferometer to detect the vibration amplitude. This technique is rather simple: the optical path to the substrate is compared with a reference path. The interferometer provides a sensitivity of about $0.5 \mathrm{~nm}$ with frequencies up to $100 \mathrm{kHz}$. The amplitude's absolute value can be determined directly from the interferometer signals. A disadvantage of this interferometrical method is that this technique requires an optical port which also can introduce heat from the environment. To understand the temperature dependence of the energy dissipation in solids it is also necessary to perform measurements down to the millikelvin range. Previous investigations showed that the behaviour of the test sample below $4.2 \mathrm{~K}$ can change dramatically [7]. In this temperature region heat entrance becomes more important and eliminates the possibility of using a Michelson interferometer due to the windows in the cryostat needed for the measurement. Hence, it is desirable to use a set-up being well shielded from the surroundings. One issue of our work is to test the capability of the high-performance SQUID technique for cryogenic $Q$-factor measurements. A superconducting layer on one surface of the test sample is required for the SQUIDbased read-out (figure 2). Thus, the operating temperature of this read-out technique is upwards limited to the $T_{\mathrm{C}}$ of the superconductor. For SQUID measurements an area of about $1 \mathrm{~mm}^{2}$ is sufficiently large (compared to the $\varnothing 3 \mathrm{inch}$ substrate's surface area this is only about $0.2 \%$ ). Braginski et al [8] showed that the influence of a thin layer can be neglected for the ring-down experiments. A pick-up coil is placed close to the superconducting layer. The pick-up coil and the SQUID input coil form a superconducting loop. Using a heat switch (see figure 2) a persistent current is stored in the input circuit. The flux in this loop has to be constant. Thus, a small movement of the superconducting layer causes an additional current in this loop. This current is sensitively measured by the SQUID. This measuring technique was already tested using a low-temperature dc SQUID UJ111 and was found to meet the requirements $[9,10]$. The SQUID sensor and readout electronics were developed at Jena University [11]. The 


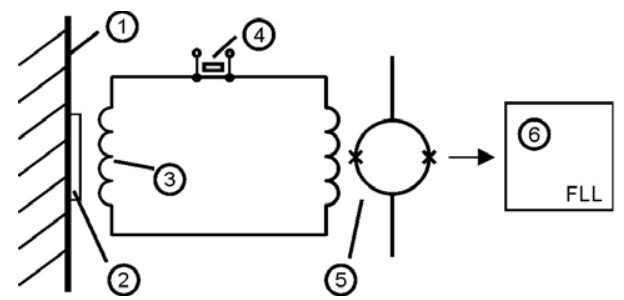

Figure 2. Scheme of the SQUID-based displacement read-out system. (1) Substrate, (2) superconducting layer (niobium, $1 \mathrm{~mm}^{2}$, thickness: $\sim 100 \mathrm{~nm}$, (3) pick-up coil, (4) read-out current injection with heat switch, (5) SQUID with input coil, (6) FLL-electronics).

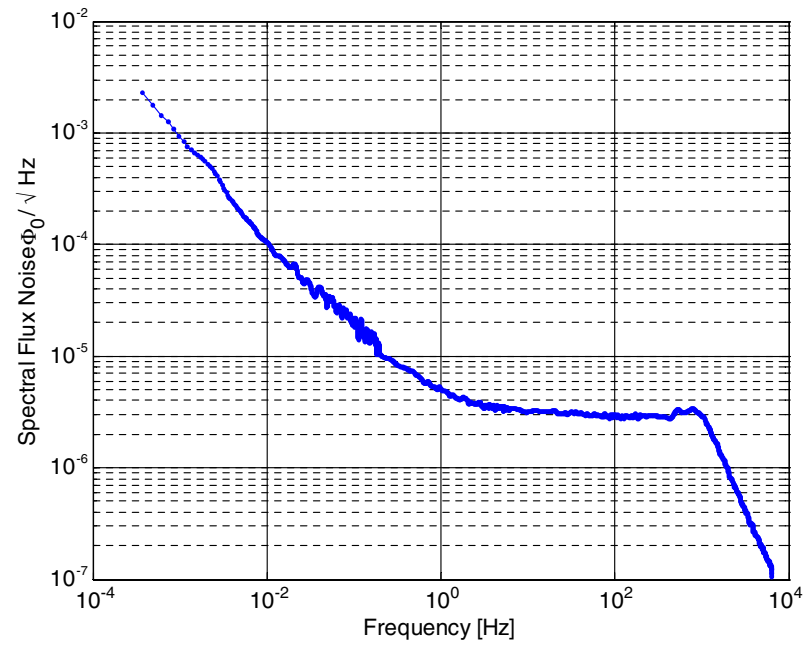

Figure 3. Spectral flux noise of the SQUID sensor UJ111 at $4.2 \mathrm{~K}$. The bandwidth of the SQUID electronics has to be increased for $Q$-factor measurements.

position resolution was mainly limited by the intrinsic SQUID noise shown in figure 3.

Considering the flux conservation of the input loop of the detector and assuming small displacements $\delta x$ the possible resolution of this measuring device was found to be $[12,13]$

$$
\delta x=\frac{2}{k} \sqrt{\frac{L_{I}}{L_{\mathrm{SQ}}}} \frac{1}{I} \frac{1}{\alpha} \delta \Phi_{\mathrm{SQ}},
$$

where $k$ is the coupling constant between input coil and SQUID loop, $L_{I}$ and $L_{\mathrm{SQ}}$ are the input coil's and SQUID's inductance, $I$ is the current in the read-out circuit, $\alpha$ is the slope of the inductance-displacement transfer function, and $\delta \Phi_{\mathrm{SQ}}$ is the flux noise of the SQUID. With typical parameters for a SQUID system using our SQUID UJ111 [11], e.g. $k=0.9, L_{I}=$ $0.8 \mu \mathrm{H}, L_{\mathrm{SQ}}=40 \mathrm{pH}, \delta \Phi_{\mathrm{SQ}}=5 \times 10^{-6} \Phi_{0} \mathrm{~Hz}^{-1 / 2}$, $I=100 \mathrm{~mA}$, and $\alpha=0.1 \mathrm{nH} \mu \mathrm{m}^{-1}$, the displacement spectral noise density is $\delta x=3 \times 10^{-13} \mathrm{~m} \mathrm{~Hz}^{-1 / 2}$. This exceeds the sensitivity given by the Michelson interferometer in use (approximately $0.5 \mathrm{~nm}$ at $100 \mathrm{kHz}$ bandwidth).

\section{Results}

Different measurements have been done during commissioning of the cryostat. To test and characterize the new cryogenic setup crystalline quartz and silicon were used as test materials. First the eigenfrequencies of the test samples were determined.

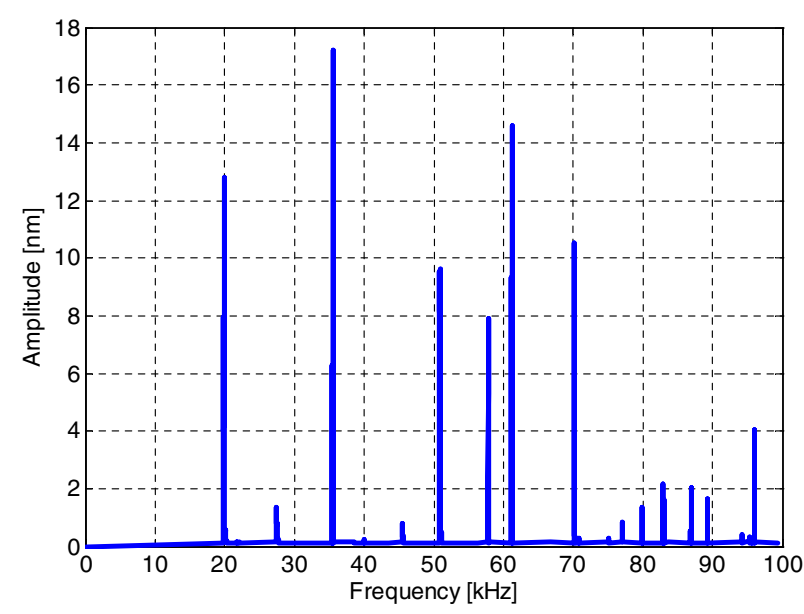

Figure 4. Results of a mode scan of a crystalline quartz test sample (diameter $76 \mathrm{~mm}, d=24 \mathrm{~mm}$, tungsten wire (diameter $50 \mu \mathrm{m}$ ) suspension) at $300 \mathrm{~K}$. In the frequency range from 10 to $100 \mathrm{kHz}, 13$ modes were detected. One scan from 10 to $100 \mathrm{kHz}$ lasts about $12 \mathrm{~h}$ with a frequency step of $2 \mathrm{~Hz} \mathrm{~s}^{-1}$.

This was assisted by a FEA based on numerical modal analysis using the software package ANSYS to give an estimate of the eigenfrequencies. The test mass model was meshed into about 15000 hexahedra with anisotropic material constants to meet the mechanical properties of the samples. Figures 4 represents the results of a mode scan of a crystalline quartz test sample.

In the frequency range from 10 to $100 \mathrm{kHz}, 13$ modes could be observed. Afterwards, the eigenfrequencies were determined more precisely by an additional scan with smaller frequency steps. Then the $Q$-factor of selected modes was investigated. Therefore the certain mode was excited. When the amplitude $a$ reaches an observable value the exciter is switched off and the free decaying vibration is recorded (figure 5). It follows an exponential law:

$$
a=a_{0} \cdot \mathrm{e}^{-t / \tau}
$$

where $a_{0}$ is the initial amplitude and $\tau$ the characteristic ringdown time of the system (the amplitude decays to $1 / \mathrm{e}$ of the initial value of the amplitude). The $Q$-factor-as a measure of internal dissipation-is defined as the ratio between the energy $E$ stored in a vibrating system and the energy loss $\Delta E$ per cycle of oscillation:

$$
Q=2 \pi \cdot \frac{E}{\Delta E}
$$

Within the approximation of a low loss system, equation (3) can be rewritten as [8]

$$
Q=\pi \cdot f_{0} \cdot \tau
$$

with the eigenfrequency $f_{0}$ of the test body and the ringdown time $\tau$ which is obtained from ring-down experiments. Figure 5 gives a comparison of the ring-down time of an eigenmode of a crystalline quartz sample at two different temperatures. By cooling down from 300 to $6.7 \mathrm{~K}$ the $Q$ factor increases from $3.0 \times 10^{6}$ to $3.3 \times 10^{7}$. This measurement was done at different temperatures with both crystalline quartz and silicon. The dependence of the measured $Q$-factor on 


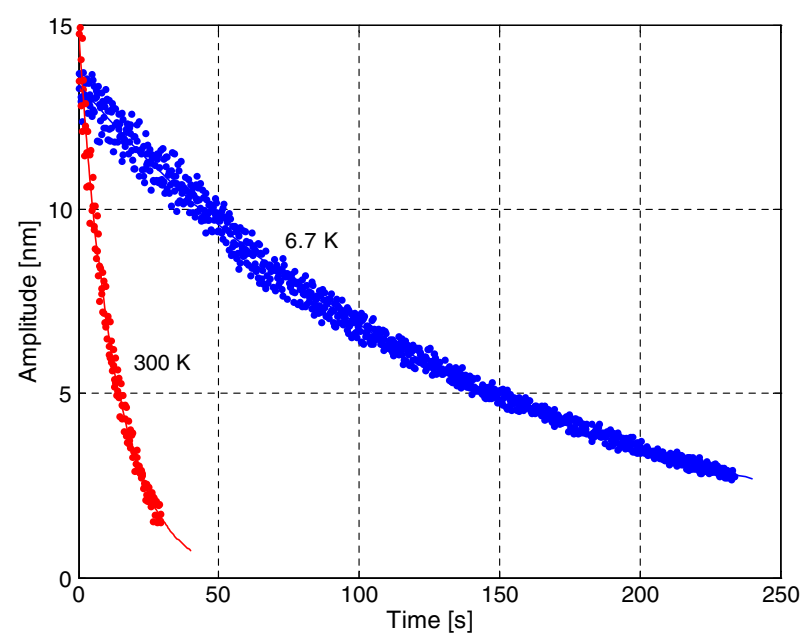

Figure 5. Free decaying ring-down of an eigenmode of a crystalline quartz substrate (diameter $76 \mathrm{~mm}, d=24 \mathrm{~mm}$, resonant frequency: $70.8 \mathrm{kHz}, 50 \mu \mathrm{m}$ tungsten wire suspension) at 300 and $6.7 \mathrm{~K}$. The $Q$-factor of that mode of $3.0 \times 10^{6}$ at $300 \mathrm{~K}$ and $3.3 \times 10^{7}$ at $6.7 \mathrm{~K}$ was achieved. The higher read-out noise at low temperatures corresponds to residual signals of other modes due to the high $Q$-factors and is not related to a higher thermal noise. Optical interferometric read-out was used.

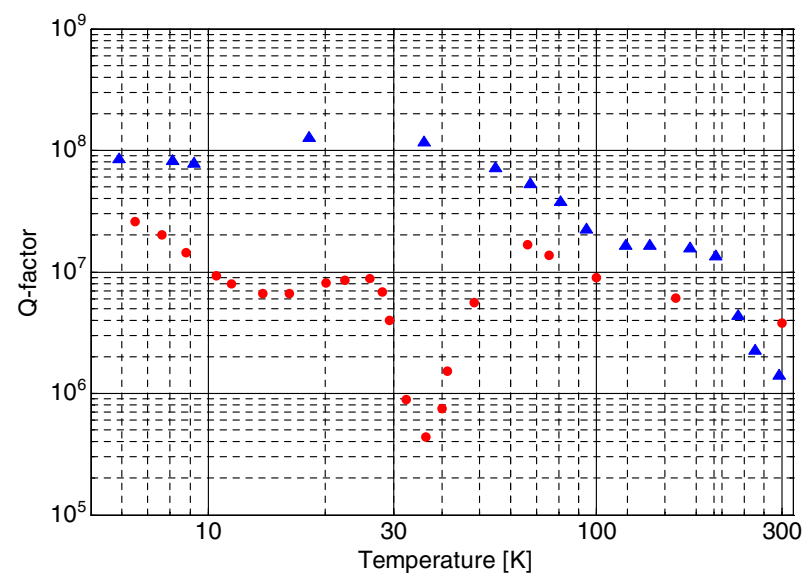

Figure 6. Dependence of the $Q$-factor on the temperature of crystalline quartz and silicon $\boldsymbol{\Delta}$. Both samples have a cylindrical shape with a diameter of $76 \mathrm{~mm}$ and a thickness of $24 \mathrm{~mm}$. All surface areas are polished to an optical quality better $\lambda / 4$.

the temperature is given in figure 6. At $37 \mathrm{~K}$ the $Q$ factor of crystalline quartz decreases due to impurities in the crystal [14]. These impurities distort the lattice and thus introduce an alternative equilibrium position with a slightly different energy of the oxygen atoms creating a two-level system. Transitions occur if the energy difference matches the thermal energy. In contrast, silicon shows an increasing $Q$ factor with decreasing temperature down to $18 \mathrm{~K}$. Below $18 \mathrm{~K}$ the $Q$-factor decreases slightly.

\section{Conclusion}

A special cryogenic system for mechanical $Q$-factor measurements of test bodies was built. It allows the investigation of the $Q$-factor dependence on temperature in the range 5-300 K. Two possible read-out mechanisms were presented. First measurements with the optical interferometric read-out were done. On a silicon substrate $Q$-factors greater than $10^{8}$ were demonstrated.

The SQUID-based read-out technique is an appropriate method for increasing the sensitivity of the measuring system at low temperatures. Furthermore it opens the usability of the ring-down-based $Q$-factor measurements at temperatures well below $4.2 \mathrm{~K}$.

The cryogenic investigations of the dependence of the $Q$ factor of optical components on the temperature contribute to the enhancement of the sensitivity of gravitational wave detectors as well as to the improvement of knowledge of the dissipation mechanism in solids.

\section{Acknowledgments}

This work was supported in part by the German DFG (Deutsche Forschungsgemeinschaft) under contract SFB Transregio 7. The authors would like to thank the GEO600 team of Karsten Danzmann as well as the Jena University Microoptics group of Andreas Tünnermann for their helpful discussions.

\section{References}

[1] Abbott B et al 2004 Detector description and performance for the first coincidence observations between LIGO and GEO Nucl. Instrum. Methods Phys. Res. A 517 154-79

[2] Uchiyama T et al 2004 Present status of large-scale cryogenic gravitational wave telescope Class. Quantum Grav. 21 S1161-72

[3] Nietzsche S, Zimmer A, Vodel W, Thürk M, Schmidl F and Seidel P 2004 Proposed cryogenic $Q$-factor measurement of mirror substrates Class. Quantum Grav. 21 S1133-8

[4] Sun K-X and Byer R L 1998 All-reflective Michelson, Sagnac, and Fabry-Perot interferometers based on grating beam splitters Opt. Lett. 23 567-9

[5] Bunkowski A, Burmeister O, Beyersdorf P, Danzmann K, Schnabel R, Clausnitzer T, Kley E-B and Tünnermann A 2004 Low-loss grating for coupling to a high-finesse cavity Opt. Lett. 29 2342-4

[6] www.lakeshore.com

[7] Classen J, Rohr I, Enss C, Hunklinger S and Laermans C 1999 Low frequency elastic properties of neutron-irradiated quartz. Comparison with glasses Eur. Phys. J. B 10 623-33

[8] Braginski V B, Mitrofanov V P and Panov V I 1985 Systems with Small Dissipation (Chicago, IL: University of Chicago Press)

[9] Nietzsche S $2001 P h D$ Thesis University of Jena (ISBN: 3-8265-9577-7)

[10] Vodel W, Nietzsche S, Neubert R and Dittus H 2002 Application of LTS-SQUIDs for testing the weak equivalence principle at the Drop Tower Bremen Physica C 372 154-7

[11] Vodel W and Mäkiniemi K 1992 An ultra low noise DC SQUID system for biomagnetic research Meas. Sci. Technol. 3 1155-60

[12] Vodel W, Koch H, Nietzsche S and Zameck Glyscinski J 1997 Low noise LTS SQUIDs for application in gravitational experiments IEEE Trans. Appl. Supercond. 7 3343-6

[13] Dittus H, Greger R, Lochmann S, Vodel W, Koch H, Nietzsche S, Zameck Glyscinski J, Mehls C and Mazilu P 1996 Testing the weak equivalence principle at the Bremen drop tower: report on recent developments Class. Quantum Grav. 13 A43-51

[14] Bömmel H E, Mason W P and Warner A W 1956 Dislocations, relaxations, and anelasticity of crystal quartz Phys. Rev. 102 64-71 